\title{
Front Matter: Volume 11053
}

, "Front Matter: Volume 11053," Proc. SPIE 11053, Tenth International Symposium on Precision Engineering Measurements and Instrumentation, 1105301 (7 March 2019); doi: 10.1117/12.2529589

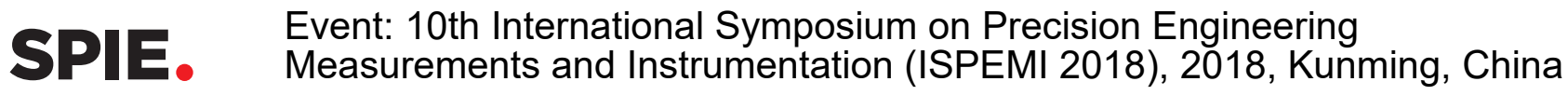




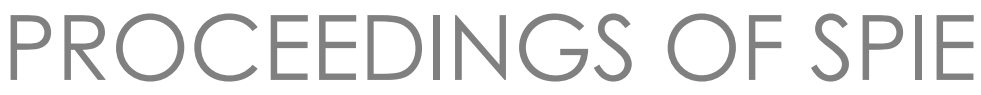

\title{
Tenth International Symposium on Precision Engineering Measurements and Instrumentation
}

\author{
Jiubin Tan \\ Jie Lin \\ Editors
}

\section{8-10 August 2018}

Kunming, China

Organized by

International Committee on Measurements and Instrumentation (ICMI) • Instrumentation Committee of CSM (IC-CSM) • Harbin Institute of Technology (HIT)

Sponsored by

International Committee on Measurements and Instrumentation (ICMI) - National Natural Science Foundation of China (NSFC) • Chinese Society for Measurement (CSM) • China Instrument and Control Society (CIS)

\section{Cooperating Organizations}

Kunming University of Science and Technology (KUST) • Beijing Information Science and Technology University (BISTU) - Hefei University of Technology (HFUT) • Electricity and Magnetism Committee of CSM (EMC-CSM)

Published by

SPIE

\section{Volume 11053}

Part One of Two Parts 
The papers in this volume were part of the technical conference cited on the cover and title page. Papers were selected and subject to review by the editors and conference program committee. Some conference presentations may not be available for publication. Additional papers and presentation recordings may be available online in the SPIE Digital Library at SPIEDigitallibrary.org.

The papers reflect the work and thoughts of the authors and are published herein as submitted. The publisher is not responsible for the validity of the information or for any outcomes resulting from reliance thereon.

Please use the following format to cite material from these proceedings:

Author(s), "Title of Paper," in Tenth International Symposium on Precision Engineering Measurements and Instrumentation, edited by Jiubin Tan, Jie Lin, Proceedings of SPIE Vol. 11053 (SPIE, Bellingham, WA, 2019) Seven-digit Article CID Number.

ISSN: 0277-786X

ISSN: 1996-756X (electronic)

ISBN: 9781510627819

ISBN: 9781510627826 (electronic)

Published by

SPIE

P.O. Box 10, Bellingham, Washington 98227-0010 USA

Telephone +1 3606763290 (Pacific Time) · Fax +1 3606471445

SPIE.org

Copyright @ 2019, Society of Photo-Optical Instrumentation Engineers.

Copying of material in this book for internal or personal use, or for the internal or personal use of specific clients, beyond the fair use provisions granted by the U.S. Copyright Law is authorized by SPIE subject to payment of copying fees. The Transactional Reporting Service base fee for this volume is $\$ 18.00$ per article (or portion thereof), which should be paid directly to the Copyright Clearance Center (CCC), 222 Rosewood Drive, Danvers, MA 01923. Payment may also be made electronically through CCC Online at copyright.com. Other copying for republication, resale, advertising or promotion, or any form of systematic or multiple reproduction of any material in this book is prohibited except with permission in writing from the publisher. The CCC fee code is 0277$786 \times / 19 / \$ 18.00$.

Printed in the United States of America.

Publication of record for individual papers is online in the SPIE Digital Library.

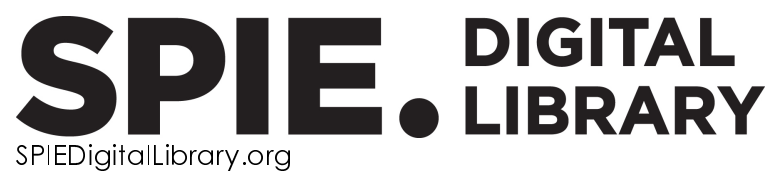

Paper Numbering: Proceedings of SPIE follow an e-First publication model. A unique citation identifier (CID) number is assigned to each article at the time of publication. Utilization of CIDs allows articles to be fully citable as soon as they are published online, and connects the same identifier to all online and print versions of the publication. SPIE uses a seven-digit CID article numbering system structured as follows:

- The first five digits correspond to the SPIE volume number.

- The last two digits indicate publication order within the volume using a Base 36 numbering system employing both numerals and letters. These two-number sets start with 00, 01, 02, 03, 04, 05, 06, 07, 08, 09, 0A, OB ... 0Z, followed by 10-1Z, 20-2Z, etc. The CID Number appears on each page of the manuscript. 


\title{
Contents
}

\author{
xiii Authors \\ xix Conference Committee \\ xxv Plenary Speaker Biographies \\ xxxvii Introduction
}

\section{Part One}

TENTH INTERNATIONAL SYMPOSIUM ON PRECISION ENGINEERING MEASUREMENTS AND INSTRUMENTATION

1105302 Development of optic-electronic autocollimators for monitoring the angular displacements of large objects (Invited Paper) [1 1053-137]

1105303 Development of auto defect inspection system for cell phone silicone rubber gasket (Invited Paper) [1 1053-1]

1105304 Review of the opaque coolant barrier removal techniques for in-process form profile optical measurement (Invited Paper) [11053-206]

$1105305 \quad$ Holographic fabrication of two-dimensional scale gratings for surface encoder by using an orthogonal type two-axis Lloyd's mirror interference lithography (Invited Paper) [1 1053-156]

1105306 Autonomous time synchronization method of wireless ad hoc sensor network and its implementation on CC1350 system [1 1053-177]

1105307 Multi-matrix optic-electronic systems for measuring the line shifts of the points on the radio-telescope main mirror (Invited Paper) [1 1053-138]

1105308 All-optical difference engine for in-process defect inspection for roll-to-roll printed electronics (Invited Paper) [1 1053-19]

1105309 Measurement of 3-dB linewidth of FBG Fabry-Perot interferometer using tunable fiber laser (Invited Paper) [11053-11]

11053 OA Sensing the atmosphere of coastal areas of laser detection methods [1 1053-84]

$11053 \mathrm{OB} \quad$ Research of the polarization-optical parameters of a solid-state matrix photomultiplier [11053-15]

11053 OC A fast infrared thermal imaging detection method based on spatial correlation (Invited Paper) [11053-17] 
11053 OD Research and application of a novel randomly encoded hybrid grating interferometric wavefront sensor (Invited Paper) [1 1053-13]

11053 OE Energy analysis method of the laser tracing measurement optical system (Invited Paper) [1 1053-27]

11053 OF Precision assessment of high-speed railway slab intelligent inspection system (Invited Paper) [1 1053-30]

11053 OG Equivalent circuit model based on the average period for periodic overlapping circle metallic mesh (Invited Paper) [1 1053-33]

$11053 \mathrm{OH} \quad$ Process optimization: internal feature measurement for additive-manufacturing parts using x-ray computed tomography (Invited Paper) [1 1053-40]

$11053 \mathrm{Ol} \quad$ Statistical measurement method of the standard particles through airborne particle counter based on FESEM (Invited Paper) [1 1053-55]

11053 0J Method for high-precision distance estimation and dispersion mismatch compensation in frequency scanning interferometry (Invited Paper) [1 1053-74]

11053 OK Development of surface profile measurement system based on super luminescent diode light source (Invited Paper) [1 1053-79]

$11053 \mathrm{OL} \quad$ The optimization of segment's axial support point for large astronomical telescopes (Invited Paper) [1 1053-81]

11053 OM Multiple-exposure adaptive selection algorithm for high dynamic range 3D fringe projection measurement (Invited Paper) [1 1053-92]

11053 ON Applications of wavefront modulation devices in aspheric and freeform measurement (Invited Paper) [11053-100]

1105300 Dual-wavelength off-axis quasi-common path digital holography using polarization multiplexing and flipping (Invited Paper) [1 1053-105]

11053 OP Micro-LED optical engine with biologically inspired artificial compound eyes for picoprojection display (Invited Paper) [1 1053-115]

$110530 Q \quad$ Real-time 3D shape measurement by fringe projection and GPU parallel computing (Invited Paper) [11053-148]

11053 OR A new non-contact coordinate measuring machine equipped with light-duty optical probe based on fringe projection profilometry (Invited Paper) [11053-164]

11053 OS The measurement technology for precision peg-in-hole assembly (Invited Paper) [1 1053-183]

11053 OT A novel breakpoint detection method based on dynamic programming for linear laser scanner (Invited Paper) [1 1053-205]

11053 OU Establishment of standard device for high rotational speed generation (Invited Paper) [1 1053-207] 
$11053 \mathrm{OV} \quad$ A two-probe linear encoder by using an arrayed scale grating stitched by multiple separate short gratings [11053-166]

11053 OW GMA internal magnetic field measurement based on FBG [11053-78]

11053 0X Development of hybrid measuring system for the complex micro-arrayed surface [11053-7]

11053 OY Error analysis of target trajectory tracking applied for measurement of high speed spindle [11053-3]

$110530 Z$ Online vision system for battery FPC connector defect detection based on active shape model template matching [11053-35]

1105310 Misalignment recognition of mass pan in joule balance [1 1053-23]

1105311 The possibility of measuring low altitudes above the sea surface with pulsed laser altimeter under conditions of fog [1 1053-213]

1105312 Improved design principle of Dyson concentric infrared imaging spectrometer [11053-217]

1105313 Two-dimensional reflective optical encoder based on point source illuminated grating imaging [11053-44]

1105314 Research of the temperature influence on the error rate of incremental optical-electronic encoders of linear displacements based on raster structures [1 1053-222]

1105315 A domain adaptation deep transfer method for image classification [1 1053-5]

1105316 Control circuit design of magnetic suspension stylus measuring instrument [1 1053-26]

1105317 Optimization on metering accuracy of smart electricity meter by temperature compensation [11053-6]

1105318 Study on temperature characteristics of triangular prisms ring cavity [1 1053-8]

1105319 Construction of a compact laser wavemeter with compensation of laser beam drift [1 1053-9]

$110531 \mathrm{~A} \quad$ An integrated linear time-grating displacement sensor with single alternating light field [11053-10]

11053 1B Laser frequency locking system using orthogonally demodulated Pound-Drever-Hall method [11053-12]

11053 1C Dynamic sensitivity distribution of linear electrostatic sensor matrix [1 1053-16]

11053 1D An exchangeable end effector for multi-part-assembly system [1 1053-18]

$110531 \mathrm{E} \quad$ Using carbon nanotube membrane as counter electrode in voltammetric electronic tongue system [1 1053-20] 
11053 1F Analysis of contacting characteristics on aerostatic bearing stylus displacement sensor [1 1053-22]

$110531 \mathrm{G}$ Synthesis and metrology of cellulose nanocrystal films [1 1053-24]

$110531 \mathrm{H} \quad$ An interrogation method with high resolution and high response speed for FBGs-based sensors [1 1053-25]

$1105311 \quad$ Combining compound eyes and human eye: a hybrid bionic imaging method for FOV extension and foveated vision [1 1053-31]

$110531 \mathrm{~J} \quad$ Bionic vision improves the performances of super resolution imaging [1 1053-32]

$110531 \mathrm{~K} \quad$ Robust concrete crack recognition based on improved image segmentation and machine learning [1 1053-34]

$11053 \mathrm{lL} \quad$ Complicated intermittent scratches detection research on surface of optical components based on adaptive sector scanning algorithm cascading mean variance threshold algorithm [1 1053-36]

$110531 \mathrm{M} \quad$ Characterization of beam splitter using Mueller matrix ellipsometry [1 1053-48]

11053 1N A chi-square statistics of arithmetic mean and applications to inter-laboratory comparison [1 1053-37]

1105310 Extracting road edges from MLS point clouds via a local planar fitting algorithm [1 1053-39]

11053 1P A novel signal process system for angular displacement sensor of time-grating [11053-41]

$110531 Q \quad$ Characterization of a liquid crystal variable retarder by Mueller matrix ellipsometry [1 1053-42]

11053 1R Defect detection method for complex surface based on human visual characteristics and feature extracting [1 1053-46]

11053 is Micro Coordinate Measurement Machine ( $\mu \mathrm{CMM})$ using voice coil actuator with interferometric position feedback [1 1053-49]

$110531 \mathrm{1T}$ A measurement method for probe microsphere of micro-CMM with double SPMs [1 1053-50]

$110531 \mathrm{U} \quad$ Aerosol particle size distribution retrieval algorithm and error analysis based on multiwavelength radar [1 1053-51]

11053 1V Investigation on the dynamic characteristics of CG-6 relative gravimeter for the micro-gravity network [1 1053-52]

11053 IW Optical fiber Fabry-Perot acoustic sensor based on large PDMS diaphragm [1 1053-56]

11053 1X A research on bolt loosening monitoring based on Lamb wave [1 1053-57] 
$110531 Y$ Development of in-situ measuring geometrical characteristics method for crankshaft [1 1053-63]

1105312 A novel miniaturized capacitive absolute angular position sensor based on time-grating with reflective structure [11053-64]

1105320 The analysis of key comparison reference value and its uncertainty using Markov chain Monte Carlo method [11053-53]

1105321 Deformation measurement of testing machine based on laser interference method [1 1053-66]

$1105322 \quad$ Corrected differential fitting for height extraction in chromatic confocal microscopy [1 1053-67]

$1105323 \quad$ Evaluation of task specific measurement uncertainty for gear measuring instrument using VGMI [1 1053-68]

1105324 Dual-comb generation from a dual-ring hybrid mode-locked fiber laser [1 1053-70]

1105325 Critical point determination method of thermal-force coupling deformation of CNC machine tool bed [1 1053-72]

1105326 Soil moisture sensor design based on fiber Bragg grating [1 1053-75]

$1105327 \quad$ Kinematics identification and measurement accuracy verification of articulated arm coordinate measuring machines [11053-77]

1105328 Self-calibration method of precision shafting angle measurement error based on multiple reading heads [11053-80]

1105329 Theoretical analysis and digital simulation of a new capacitive sensor [1 1053-82]

$110532 \mathrm{~A} \quad$ Error mapping for rotary axes of machine tools based on pose measurement principle [1 1053-83]

$110532 \mathrm{~B} \quad$ Method of squareness measurement based on laser alignment measuring system [1 1053-85]

$110532 \mathrm{C} \quad$ Super-resolution scanning microscopy with virtually structured illumination [1 1053-86]

11053 2D Angle measurement for cross-line target image based on fourier-polar transform algorithm [1 1053-87]

$110532 \mathrm{E} \quad$ Microfluidic contact lenses for continuous non-invasive intraocular pressure monitoring [1 1053-88]

$110532 \mathrm{~F} \quad$ Eye positioning based on windowed gray-scale integral projection algorithm [1 1053-89] 


\section{Part Two}

$110532 \mathrm{G}$ A novel Earth's gravity method for accelerometer calibration [1 1053-93]

$110532 \mathrm{H} \quad$ Self-calibrating phase shifting shadow moiré technique [1 1053-94]

$1105321 \quad$ Research on key technologies of quadrupole electromagnetic tweezer [11053-96]

$110532 \mathrm{~J} \quad$ Error analysis method of weighing cycles based on robotic mass measurement system [11053-97]

$110532 \mathrm{~K} \quad$ A high precision edge detection method for the blurred image in motion measurement [11053-98]

$110532 \mathrm{~L} \quad$ A filter algorithm based on ARMA model to suppress the influence of atmospheric disturbance in laser straightness measurement [1 1053-99]

$110532 \mathrm{M}$ Torque standard machine for calibration of reference torque wrench and torque transducer at Nim [1 1053-102]

$110532 \mathrm{~N} \quad$ Strain transfer characteristics of resistance strain-type transducer [1 1053-103]

1105320 Design of flexure hinges in an H-drive precision stage with a large span [1 1053-104]

$110532 \mathrm{P}$ Investigation on gPhone gravimeter-119 for gravity variations observation during the 10th International Comparison of Absolute Gravimeters (ICAG-2017) [11053-106]

$110532 \mathrm{Q}$ Multi-structure elements morpholoy for improved anti-noise edge detection [1 1053-107]

$110532 R \quad$ Analysis of the extraction accuracy of the corner point of the camera using polarization imaging [1 1053-108]

$110532 S \quad$ Evaluation of the diffraction correction during the 10th International Comparison of Absolute Gravimeters (ICAG-2017) [1 1053-110]

$110532 \mathrm{~T} \quad$ Improved two dimensional micro-/nanoradian angle generator with single rotation center located on tilting plane and error compensation of capacitive sensors [1 1053-1 11 ]

$110532 U$ Dependence of micro-morphology of electrical strain gauges on stress distribution [11053-114]

$110532 \mathrm{~V} \quad$ Research and evaluation of geometric element data fitting software for coordinate measurement machine [11053-117]

11053 2W Comparison of spacing detection algorithms for optical straight fringes images [11053-118]

$110532 X \quad$ Phase measuring method and error compensation in 3D profile measurement [11053-119]

$110532 Y \quad$ A design of high-accuracy angle measurement system for satellite AIT processing [1 1053-120] 
$110532 Z$ FPGA-accelerated one-dimensional Fourier reconstruction LCD defect detection algorithm [11053-121]

$1105330 \quad$ Keysight B1505A power device analyzer output pulse current calibration method [11053-122]

$1105331 \quad$ Review of convolutional neural network optimization and training in image processing [1 1053-125]

1105332 A passively Q-switched dual-frequency Nd:YAG laser for SBS generation and amplification [1 1053-127]

1105333 Effect of varying load on angle measurement deviation of rotary table [11053-128]

1105334 Measurement method of the compression coefficient of near-monocrystalline silicon density liquid [11053-129]

1105335 Research on omnidirectional motion of AGV based on vision guidance [1 1053-135]

1105336 Measurement of deionized water density based on single silicon sphere [11053-130]

$1105337 \quad$ Non-destructive rapid inspection methods for spatial light modulator using swept source optical coherence tomography [11053-131]

1105338 More efficient optical sectioning structured illumination microscopy [1 1053-133]

1105339 Probe error analysis of articulated arm coordinate measuring machine [1 1053-134]

11053 3A Parameter identification of inertial velocity sensor for low-frequency vibration measurement [1 1053-136]

11053 3B Design, fabrication, identification and test of a closed-loop moving magnetic scanning module for RGB laser projector [11053-139]

11053 3C Force control and visual measurement in precision assembly [1 1053-142]

11053 3D Adaptive segmentation method of pressed character image based on Wellner algorithm [11053-143]

11053 3E Research on calibration method of rotating axis in line structure light measurement system [1 1053-144]

11053 3F Decoupling atmosphere Rayleigh-Brillouin scattering spectrum in kinetic regime [1 1053-145]

11053 3G Automatic real-time compensation of wavelength of heterodyne interferometer [1 1053-146]

$110533 \mathrm{H} \quad$ A real-time nonlinear error measurement method with picometer accuracy and free from target motion state [11053-147] 
$1105331 \quad$ Design and testing of a chromatic dispersion system for displacement application by using a spatial-bandpass-filter [11053-149]

11053 3J Instrument and equipment monitoring system based on the internet of things technology [1 1053-150]

11053 3K Directional phase-shift circular arrays targets for out-of-focus camera calibration [11053-140]

11053 3L Accurate measurement of displacement using acceleration signal [1 1053-151]

11053 3M Development of portable digital ultrasonic guided wave detector based on COM Express [1 1053-152]

11053 3N A measuring method of coaxiality errors for apart axis [1 1053-153]

$1105330 \quad$ Fabrication process and measurement of injection molding aspheric lens [11053-154]

11053 3P A compact design of optical scheme for a two-probe absolute surface encoders [11053-155]

$110533 Q \quad$ Wavelength calibration system for diode laser [1 1053-157]

$110533 R \quad$ Choice of optimal resolution and array for integrated photosynthetically active radiation spectroradiometer [1 1053-159]

$1105335 \quad$ Characterization of surface roughness by double blanket model from laser speckle images [1 1053-160]

11053 3T Embedded intelligent camera algorithm based on hardware IP [1 1053-162]

$110533 \mathrm{U} \quad$ Detection of ring gear surface defects of wheel speed sensor based on neural network [1 1053-163]

11053 3V The optical-electronic autoreflection sensor for angular deformations measurement [1 1053-161]

11053 3W Design and testing of a compact optical prism module for multi-degree-of freedom grating interferometry application [1 1053-165]

11053 3X Design and implementation of flexible display reliability testing instrument [1 1053-168]

11053 3Y A real-time distance measurement data processing platform for multi-axis grating interferometry type optical encoders [11053-170]

1105332 A differential giant magnetostrictive mitro-displacement actuator [1 1053-171]

1105340 Defect detection of small modulus gear based on machine vision [1 1053-176]

$1105341 \quad$ Experimental study on non-linear calibration of two-dimensional nano-positioning stage [1 1053-178] 
1105342 Reverse compensation to the angle estimate error using multi-groups sensor [11053-179]

1105343 Precise measurement of large roll angle using digital speckle pattern interferometry [1 1053-180]

$1105344 \quad$ Ultrasonic characteristics of contact stress of small interference fitting parts [11053-182]

1105345 Bias electric field distribution analysis based on finite difference method with non-uniform grids for a non-contact tunneling current probe [1 1053-184]

1105346 An enhanced homodyne laser vibrometer based on an auto-gain module [11053-185]

1105347 Research on multi-degree-of-freedom and high-precision touch screen characteristic test instrument [11053-169]

$1105348 \quad$ Ultra-precision temperature control of circulating cooling water based on fuzzy-PID algorithm [1 1053-186]

1105349 An identification method for casing weld in complex environment [1 1053-187]

11053 4A Image displacement analysis for electro-optical system for deflection measurement of floating docks [11053-188]

11053 4B Design and simulation of a 2-DOF parallel linear precision platform utilizing piezoelectric impact drive mechanism [1 1053-189]

11053 4C Parameter optimization of measuring and control elements in the monitoring systems of complex technical objects with triple reflector [1 1053-190]

11053 4D The meat product quality control by a polarimetric method [11053-191]

$110534 \mathrm{E} \quad$ Online test method and standard instruments for verification of traffic speed meters based on actual traffic [1 1053-193]

$110534 \mathrm{~F} \quad$ Field test method and standard instruments for verification of traffic speed meters based on test vehicle [1 1053-194]

11053 4G Design and performance analysis of a novel thermos-structure for measuring thermal drift of optics in a next generation interferometer [1 1053-195]

$110534 \mathrm{H} \quad$ Choosing parameters of active reference mark optical-electronic systems spatial position control [11053-196]

110534 C $\quad$ CMC based on unitary linear regression equation [11053-197]

11053 4J Method for spherical form error evaluation using cuckoo search algorithm [1 1053-199]

11053 4K Design of Gaussian filters based on odd and even functions used for non-closed circular profile [1 1053-200] 
$110534 \mathrm{~L} \quad$ Research of variable-frequency big current calibration [1 1053-201]

$110534 \mathrm{M} \quad$ Long-range automatic precision displacement measuring of winding system using double timing belt transmission [1 1053-202]

$110534 \mathrm{~N} \quad$ A uniform and flexible model for three-dimensional measurement of line-structured light sensor [11053-203]

1105340 Subwavelength focusing and experimental detection of large-scale metallic multi-annular metasurfaces [1 1053-208]

11053 4P The internal air gap measurement equipment for dynamic pressure motor [1 1053-209]

$110534 Q \quad$ Study on resonant high-acceleration calibration system [1 1053-211]

11053 4R Design of optical accelerometer using four-quadrant photodetector [1 1053-212]

1105345 A novel two-dimensional inductive sensor based on planar coils [1 1053-218]

$110534 \mathrm{~T} \quad$ Establishment of measuring coordinate system for large gears measurement by using gauge blocks [1 1053-221]

$110534 \mathrm{U} \quad$ Equivalent thin film model with complex refractive index of resonant micromesh [1 1053-223]

$110534 \mathrm{~V} \quad$ System design of lithium battery internal resistance measurement using LabVIEW [1 1053-224] 


\section{Authors}

Numbers in the index correspond to the last two digits of the seven-digit citation identifier (CID) article numbering system used in Proceedings of SPIE. The first five digits reflect the volume number. Base 36 numbering is employed for the last two digits and indicates the order of articles within the volume. Numbers start with 00, 01, 02, 03, 04, 05, 06, 07, 08, 09, OA, OB...0Z, followed by 10-12, 20-2Z, etc.

\begin{tabular}{|c|c|}
\hline An, Feiyue, $3 M$ & Chen, Shaowei, 3D \\
\hline An, Hongbin, $2 \mathrm{E}$ & Chen, Xiuguo, $1 Q$ \\
\hline An, Jin, OY & Chen, Xue, OC \\
\hline An, Jiong, OF & Chen, Yalu, $2 Y$ \\
\hline Baev, S. S., 3R & Chen, YU, 15 \\
\hline Bai, Fuzhong, 2D, 2W & Cheng, Biyao, 40 \\
\hline Bai, Jiao, 31 & Cheng, Fang, $\mathrm{OH}$ \\
\hline Bai, Jie, OU, 4E, 4F & Cheng, Jiachun, $4 Q$ \\
\hline Bai, Libing, $0 \mathrm{C}$ & Cheng, Xiang, $3 Q$ \\
\hline Bai, Yang, 10 & Cheng, Xiaosheng, OM \\
\hline Bao, Jian, 35 & Cheng, Xuemin, 31,30 \\
\hline Bao, Wenhui, 28 & Cheng, Yuhua, OC \\
\hline Bao, Xue-Song, 36 & Chung, Chien-Kai, 3B \\
\hline Bian, Dian, OK & Cui, Haihua, OM \\
\hline Bian, Xiaoyun, 18 & Cui, Jianjun, 21 \\
\hline Bian, Xingyuan, 45 & Cui, Jiwen, $1 \mathrm{H}, 2 \mathrm{Q}, 4 \mathrm{~N}$ \\
\hline Blokhina, Anastasia A., 14,4C, 4D, $4 \mathrm{H}$ & Cui, Junning, 45,48 \\
\hline Bütefisch, Sebastian, is & Cui, Xuewei, 2V \\
\hline Cai, Chenguang, $2 \mathrm{G}, 2 \mathrm{~K}, 4 \mathrm{Q}$ & Dai, Tianliang, 4B \\
\hline Cai, Jianzhen, $4 \mathrm{~L}$ & Dang, Hong, $1 \mathrm{H}$ \\
\hline Cai, Yindi, 19 & Danzebrink, Hans-Ulrich, is \\
\hline Cai, Zeliang, 39 & Deng, Huiwen, $1 \mathrm{C}$ \\
\hline Cao, Cong, $1 \mathrm{G}$ & Di, Huige, $1 \mathrm{U}$ \\
\hline Cao, Jie, $11,1 \mathrm{~J}$ & Diao, Ming, 00 \\
\hline Cao, Pin, $0 D, 1 L, 1 R$ & Diao, Xiaochun, $0 Q, O R$ \\
\hline Chai, Changchun, 38, 3Q & Ding, Jiawei, 3L \\
\hline Chai, Huiting, 1L, 1R & Ding, Xiangshuai, 4J, 4K \\
\hline Chang, Cun, $4 \mathrm{~V}$ & Ding, Xuemei, 2C, 2I, 4G \\
\hline Chang, Di, 46 & Dong, Yao-hui, 32 \\
\hline Chang, Qi, 1X & Du, Hubing, $2 \mathrm{H}$ \\
\hline Chang, Qing, 4V & Du, Lei, OU, 4E, 4F \\
\hline Chang, Suping, $16,1 \mathrm{~F}$ & Du, Yubin, 1L, $1 \mathrm{R}$ \\
\hline Chang, Zhen-Xin, $4 R$ & Duan, Xili, 3D \\
\hline Chao, Xiangzhang, 4J, 4K & Fan, Bingyan, $1 \mathrm{C}$ \\
\hline Chen, Cheng, OF & Fan, Jing, 3L \\
\hline Chen, Cheng, 22 & Fan, Kuang-Chao, 19, 2L, 4R \\
\hline Chen, Hang, 13 & Fan, Zhe, OU, $4 \mathrm{E}, 4 \mathrm{~F}$ \\
\hline Chen, Hongfang, OE & Fan, Zhigang, 24 \\
\hline Chen, Honglei, $3 \mathrm{M}$ & Fang, Bo, 3L \\
\hline Chen, J. J., 3S & Fang, Chuan-Zhi, 1T \\
\hline Chen, Jiamin, OW, OY, 3A & Fang, Meiqi, 37 \\
\hline Chen, Jian, Ol & Feng, Baokai, 19 \\
\hline Chen, Jianyun, 06 & Feng, Guohua, 1L, 1R \\
\hline Chen, Jinping, OX & Feng, Jin-yang, 1V, 2P, $2 S$ \\
\hline Chen, Kai, 21 & Feng, Kunpeng, $1 \mathrm{H}$ \\
\hline Chen, Lei, OZ & Feng, Xiaobing, 08 \\
\hline Chen, Liangzhou, $\mathrm{OL}, 2 \mathrm{E}$ & Flügge, Jens, 1S \\
\hline Chen, Li-Juan, $1 T$ & Fu, Haijin, 24, 3H, 46, 4G \\
\hline Chen, Ming-Fu, 3B & Fu, Jian, 22 \\
\hline
\end{tabular}


Fu, Min, 1 A

Fu, Xing, OX

Fu, Yanjun, 3E

Gao, Fei, $0 Z$

Gao, Guanbin, 27

Gao, Heming, 1C, $1 \mathrm{X}$

Gao, Sitian, 3G, 41

Gao, Xianming, 2A

Gao, Xiaojuan, 2W

Gao, Y., 04

Gao, Yue, 13

Gao, Zhiliang, ol

Gorbachev, Alexey A., 4A

Grishkanich, Alexander, OA

GU, Honggang, $1 Q$

Gu, Yuling, 33

Guo, Beichen, 30, 41

Guo, Jiahao, 24

Guo, Junjie, 2A

Guo, Pan, 3T

Guo, Tong, OX

Guo, Xiaopeng, 37

Han, Liling, 4B

Hang, Chenzhe, 1N

Hao, Qun, ON, 11, 1 J, 30

$\mathrm{HaO}$, Zhonghan, $4 \mathrm{~V}$

Happonen, Tuomas, 08

$\mathrm{He}$, Qing, 10

$\mathrm{He}$, Tingyao, 3F

$\mathrm{He}$, Yang, $2 X$

Ho, Chao-Ching, 03

Hoang, Anh Phuong, 4A

Hou, Haiyan, $1 \mathrm{U}$

Hou, Jia, 33

Hsu, Cheng-Chih, 13

Hu, Beibei, 30, 4l

Hu, Chunbing, 16, $1 \mathrm{~F}$

$\mathrm{Hu}$, Hongbo, OU

Hu, Jiacheng, 3L, 3U

$\mathrm{Hu}$, Jinxin, $2 \mathrm{O}$

Hu, Pengcheng, 24, 3H, 46, 4G

Hu, Xiaotang, OX

$\mathrm{Hu}, \mathrm{YaO}, \mathrm{ON}$

Hua, Dengxin, OP, 3F

Huang, Jingzhi, 4J, 4K

Huang, Linyi, 3J, 3X, 47

Huang, Lu, 41

Huang, Qiang-Xian, IT

Huang, Xiaoding, 4l, 4L

Huang, Yao, 33

Huang, Yubin, 2L

Huang, Zhenwei, $3 \mathrm{U}$

Ji, F., 3S

Ji, Qizheng, Ol

$\mathrm{Ji}$, Ruidong, $3 \mathrm{H}$

Jia, Huakun, 28

Jia, Pingping, 37

Jiang, Fei, 1B

Jiang, $\mathrm{HaO}, 1 \mathrm{M}, 1 \mathrm{Q}$

Jiang, Hongzhi, $\mathrm{OQ}, \mathrm{OR}$
Jiang, Jiabin, 1L, 1R

Jiang, Lin, 4J, 4K

Jiang, Xiangqian, 22

Jiao, Ming-xing, 18, 1B, 32

Jin, Hui, OG

Jin, Peng, OY, 13

Jin, Wei, $3 G$

Jin, Yihua, $1 \mathrm{H}$

Kang, Jiale, 3F

Kang, Yanhui, 2B

Kleshchenok, Maksim A., 14, 4C, 4D, 4H

Kong, Jun, 2D

Konyakhin, Igor A., 02, 07, OA, OB, 14, 3R, 3V, 4A, $4 \mathrm{C}, 4 \mathrm{D}, 4 \mathrm{H}$

Korotaev, Valery, 3R, 4C

Kuzmin, V. N., 3R

Lai, Houhu, 2Q

Le, Jing, 3D

Leach, Richard, 08

Lebedko, E. G., 11

Lei, Dong, 2J

Lei, Liu, $3 \mathrm{U}$

Lei, Ying-Jun, 4R

$\mathrm{Li}$, Bing, $\mathrm{OZ}$

Li, Changli, $1 \mathrm{~A}$

Li, Changqi, 20

Li, Chun-jian, 1V, 2P, $2 S$

Li, Dong, 35

Li, Haitao, 2A

$\mathrm{Li}, \mathrm{HaO}, 4 \mathrm{~V}$

Li, Hong-Li, $1 T$

Li, Jiahao, 06

Li, Jingjing, 30, $4 \mathrm{I}$

$\mathrm{Li}$, Jingtao, 2C, 2

Li, Junzhong, 3A, $3 Z$

$\mathrm{Li}, \mathrm{Li}, 25$

Li, Lin, 29

Li, Qi, 3G, 41

Li, Rui-Jun, 4R

Li, Shaokang, 4T

Li, Shi, 3G, 41

Li, Shichao, OF

Li, Tong, 4M

Li, Wei, $2 X$

Li, Wei, $3 G$

Li, Weixian, 43

Li, Ximin, 29

Li, Xinghui, 05, 0V, 12, 3I, 3P, 3W, 3Y

Li, Yanwei, 1L, 1R

Li, Yawei, 3C

Li, Yazhuo, 1E

Li, Ying, 3N

Li, Yixin, $3 Z$

Li, Yongqian, 2N, $2 \mathrm{U}$

$\mathrm{Li}$, Yueqiang, $2 \mathrm{X}$

Li, Yuze, 15

Li, Zelin, $4 \mathrm{U}$

Li, Zhaojie, OM

Li, Zhe, $4 \mathrm{~N}$

$\mathrm{Li}$, Zhengkun, 10

xiv 
Li, Zhihui, $2 Y$

Liang, Jina, 3U

Liang, Zijian, OD

Liao, Wenhe, OM

Liao, Xiaosu, 2F

Lim, Pei Xian, $\mathrm{OH}$

Lin, Chen-Chang, 3B

Lin, Jie, OY, 13

Liu, Xv, 4U

Liu, Bin, 0C, 00, 1W

Liu, Bingguo, 3T

Liv, Chenghao, OQ, OR

Liu, Defeng, 3M

Liu, Dongliang, $1 Y$

Liu, Duxi, $4 M$

Liu, Enxiao, 4U

Liu, Guodong, ЗТ

Liu, Hui, 39

Liu, Ji, 43

Liu, Jiamin, $1 \mathrm{M}$

Liu, Jian, 08

Liu, Jianli, $1 \mathrm{~N}, 20$

Liu, Jianning, 18

Liu, Jingjing, $3 F$

Liu, Jun, $1 \mathrm{C}$

Liu, Jun, 1U, 26

Liu, Kang, $4 \mathrm{O}$

Liu, Lei, 00, 1W

Liu, Lingcheng, 30

Liu, Peide, 09

Liv, Qi, 40

Liu, Qiang, $4 \mathrm{O}$

Liu, Quanxin, 2R

Liu, Rongzhi, 1L, 1R

Liu, Shan-liang, 1V, $2 S$

Liv, Shiyuan, $1 M, 1 Q$

Liu, ShuJie, $2 \mathrm{~L}$

Liv, Sili, 06

Liu, Tao, 40

Liu, Wei, $3 \mathrm{M}$

Liu, Xiaojun, OL, 22, 2E, 38, 3Q

Liu, Xiaokang, $1 \mathrm{Z}$

Liu, $X U, 3 K$

Liu, $X v, 4 \mathrm{U}$

Liv, Yang, 4S

Liu, Yongmeng, 0G, 4U

Liu, Yun, 32

Liu, Zenghua, $3 \mathrm{M}$

Liu, Zhihua, 2G, 2K, 4Q

Liv, Zhirou, 4P

Lobanova, Anastasiya Y., OB

Lou, Zhifeng, OS, 3N, 44

LU, Binghui, 3T

Lu, Haiou, 05, 3W

Lu, Jhih-Jia, 03

LU, Rongsheng, 2Z, 3K, 3S

Lu, Wenlong, 22

LU, Yang, OK

Lu, Yesheng, 45, 48

Lu, Yunfeng, 10
Lu, Zheng, 18

Luan, Guangyu, 00

Luo, Jian, $3 Q$

Luo, Qiang, 1J

Luo, Qifeng, 3T

LUo, Yi, OS, 1D, 3C

Luo, Zai, 35, 39, 40

Luo, Zhi-Yong, 34

LV, Mingdong, 17

LV, Qi, 4Q

Lyu, Dajuan, 09

Ma, Guoyuan, 1N

Ma, Jiandong, 3L

$\mathrm{Ma}$, Jihui, OG

Ma, Lina, 10

Ma, Xin-yu, 34, 36

Ma, Yarui, $2 Q$

$\mathrm{Ma}$, Yifei, 09

Mao, Bin, 21

Mao, Yunlong, $2 \mathrm{U}$

Maraev, A. A., 3R

Matalaray, Thaddie, $\mathrm{OH}$

Mednikov, Sergey V., 14, 4C, 4D, 4H

Meeß, Rudolf, $1 S$

Meng, Feng, 2M

Mi, Meng, $1 T$

Min, Yingxing, 1C

Ming, Yuyang, 3D

Molnar, Gabor, 1S

Mou, Lishuang, $2 \mathrm{P}$

$\mathrm{Na}$, Jing, 27

Nekrylov, Ivan S., 14, 4C, 4D, 4H

Nguyen, D. T., 11

$\mathrm{Ni}, \mathrm{He}, 2 \mathrm{l}$

$\mathrm{Ni}$, Kai, 05, 0V, 12, 3I, 3P, 3W, 3Y

Ning, Yan, ON

Niu, Yizhao, $1 \mathrm{H}$

Ou, Jiahao, 49

Pan, Chengliang, 4B

Pan, Hao, OJ

Pan, Yinfei, $2 Z$

Pang, Yongjun, 25

Peng, Donglin, 1P, 42

Peng, Kai, 12

Peng, Tingting, $1 G$

Pu, Hongji, $1 Z$

Pu, Zhiwei, 1A

Qi, Zhou, 41

Qiao, Xiaoxu, 1D

Qin, Bo, 4P

Qin, Junfeng, 3E

Qin, Yunliang, 3L

Qin, Yuwei, 37

Qin, Ziyang, 3E

Qu, Xinghua, OJ

Ren, Lina, 18

Ren, Tongqun, OS, 4P

Ren, Yong, 31

Ryzhova, Victoria A., OA, OB, 4D

Sakhariyanova, Aiganym M., 3V 
Shan, Mingguang, 00, $1 \mathrm{~W}$

Shao, Hongwei, 21

Shao, Jiang, $1 \mathrm{~K}$

Shen, Xueping, 21

Shi, Guoqiang, $1 X$

Shi, Jian, 2T

Shi, Tangjun, $1 \mathrm{H}$

Shi, Xingrong, 2D

Shi, Yaping, 3P

Shi, YU-shu, 3G, 41

Shi, Zhaoyao, OE, $1 \mathrm{P}$

Shu, Honglin, 21

Smirnov, Leonid, OA

Song, Chang, OL

Song, Huixu, OE

Song, Yongfeng, $\mathrm{OL}$

Su, Duo-wu, 1V, 2P, $2 S$

Su, Juan, 1B

Su, Meng, 3J, 3X, 47

Su, Rong, 08

Sun, Chuanzhi, 0G, 4U

Sun, Hao, 24

Sun, Jiangong, $2 \mathrm{~A}$

Sun, $\mathrm{Na}, 26$

Sun, Qiao, OU, 4E, 4F

Sun, Shengsheng, 1D, 3C

Sun, Xun, $1 \mathrm{H}$

Sun, Zhenshan, OX

Sun, Zhi, 30

Sycheva, Elena A., $4 \mathrm{H}$

Tan, Jiubin, OG, $\mathrm{OY}, 1 \mathrm{H}, 24,2 \mathrm{O}, 2 \mathrm{Q}, 2 \mathrm{~T}, 3 \mathrm{H}, 45,46$, $4 \mathrm{~N}$

Tan, Junge, Ol

Tan, Xinran, 2T

Tang, Liang, $\mathrm{OE}$

Tang, Mingxing, 3D

Tang, Ying, $1 G$

Tang, Yuxuan, OW

Tao, Jianguo, 40

Tao, Wei, OT

Tian, Lulu, OC

Tian, Shuai, 2J

Tian, Wei, OM

Tomskiy, K. A., 3R

Tong, Lin, 36

Tong, Minh Hoa, 07

Tong, Yazhen, 4L

Tseng, Shih-Feng, 3B

Vasilev, Alexandr S., 14

Wang, Binghe, 2B

Wang, Chao-Qun, IT

Wang, Chenyan, $\mathrm{Ol}$

Wang, Dawei, 10

Wang, Faling, $2 F$

Wang, Fanyi, 1L, 1R

Wang, Ge, 10

Wang, Hewen, $1 Z$

Wang, Hui, 2Q

Wang, Jian, 13

Wang, Jian, 22
Wang, Jianhua, 23

Wang, Jiarong, 10

Wang, Jing, 3A, $3 Z$

Wang, Jin-Tao, 34, 36

Wang, Jun, OP, 3F

Wang, Ke, 46

Wang, Lei, OW, OY, 3A, 37

Wang, Linyan, 4T

Wang, LU, 17

Wang, $\mathrm{Na}, 2 \mathrm{~J}$

Wang, Peirong, 3P

Wang, Qi-yu, 1V, 2P, 2S

Wang, Sen, 27

Wang, Shaokai, 20

Wang, Shitong, 3A

Wang, Shuqiang, 30

Wang, Shuxian, 42

Wang, Tong, $4 \mathrm{O}$

Wang, Wei, $1 \mathrm{P}$

Wang, Weinong, 2V

Wang, Wen, 27

Wang, Xian, 49

Wang, Xiaodong, OS, 1D, 3C, 3N, 44, 4P

Wang, Xiaohao, 05, OV, 12, 3I, 3P, 3W, 3Y

Wang, Xinggang, OY

Wang, Xingyuan, OS, 44

Wang, Xinyun, $2 R$

Wang, Yangyang, 42, 4S

Wang, Yayong, $2 \mathrm{~L}$

Wang, Ying, 2G

Wang, Ying, 2W

Wang, Yue, OS, 3N, 44

Wang, Yue, $3 \mathrm{H}$

Wang, Yuexin, 2W

Wang, Zhigang, 2N, $2 \mathrm{U}$

Wang, Zihan, $11,1 \mathrm{~J}$

Wei, Hengzheng, 2V

Wei, Peng, 1Q

Wei, Shengyu, 3J

Werner, Christian, 1s

Wu, Bingkun, 1W

WU, Fan, 1L, 1R

Wu, Fan, 43

Wu, Guolong, 4G

Wu, Hao, 16, $1 \mathrm{~F}$

Wu, Jianwei, $4 \mathrm{~N}$

Wu, Liang, 4S

Wu, shu-qing, 1V, 2P, $2 S$

Wu, Sijin, 43

Wu, Tianjian, $4 \mathrm{~V}$

Wu, Zhiyi, 42

Xia, Guisuo, 3E

Xia, Haojie, 4B

Xiao, Chi, 2N, 2U

Xiao, Su, 3Y

Xiao, Xiang, 1L, 1R

Xiao, Xiang, 3W

Xiao, Yuqing, $1 \mathrm{~J}$

Xin, Meiting, $\mathrm{OZ}$

Xing, Jun-hong, 1B, 32 
Xing, Mingyi, $O Q, O R$

Xiong, Liangming, 09

$\mathrm{Xu}, \mathrm{Dawei}, \mathrm{OH}$

$X U$, Dinghua, $1 \mathrm{~N}, 20$

Xu, Huawei, 3J, 3X, 47

$X U$, Jingzhong, 10

$X U$, Jinshun, $4 M$

$X \cup, M . J ., 3 S$

$\mathrm{XU}$, Qianyu, 2J

$X \cup, S h i, 4 S$

$\mathrm{XU}, \mathrm{Xiangdong}, 4 \mathrm{P}$

$X U$, Yang, $O Q, O R$

$X U$, Yongxiang, 2D

$\mathrm{Xu}, \mathrm{Zhou}, 49$

Xue, Zi, 33

Yan, Hongzhou, 38, 3Q

Yan, Kejun, 1U, 26

Yan, Peng, 12

Yan, Qing, OP, 3F

Yan, Xinyu, OK

Yang, Chunling, 15

Yang, Hongtao, 25

Yang, Hongxing, 24, 3H, 46, 4G

Yang, Jing, 43

Yang, L., 3S

Yang, Lianxiang, 43

Yang, Ming, 2G, 2K

Yang, Ruitao, 24, 3H, 46

Yang, Shuming, $4 \mathrm{O}$

Yang, Tianlong, $1 \mathrm{~K}$

Yang, Wanfu, 4V

Yang, Weixi, $1 \mathrm{X}$

Yang, Wenguo, OY

Yang, WenJun, 22

Yang, Xinlong, 2A

Yang, Yongying, OD, 1L, IR

Yang, Zhijie, 12

Ye, Shuliang, 33

Ye, Xuerong, 17

Yin, Peili, 23

Yin, Xiao-qia, OT

Ying, Zhang, 2K

Yu, BO, OE

Yu, Dianhong, 29

YU, Haichao, OC, IW

Yu, Haoyong, $1 \mathrm{~J}$

$\mathrm{YU}$, Jianhong, $2 \mathrm{H}$

$\mathrm{YU}$, Liandong, $\mathrm{OK}, 28,4 \mathrm{~B}$

Yu, Liang, $1 \mathrm{~S}$

Yu, Songmin, 17

Yu, Xiaoyu, 1A

Yu, Yang, 2T

Yu, Zhicheng, $1 \mathrm{Z}$

Yuan, Weihan, 05, 0V

Zhai, Guodong, $4 \mathrm{Q}$

Zhai, Guofu, 17

Zhang, Chenchen, $2 \mathrm{~F}$

Zhang, Chi, 22, 38

Zhang, Chuanwei, 1Q

Zhang, Dian long, $2 M$
Zhang, Fanghua, 11

Zhang, Fumin, $0 \mathrm{~J}, 2 \mathrm{R}$

Zhang, Haiyun, 20

Zhang, Huan, 4I

Zhang, Jing-Yue, 36

Zhang, Lian-Sheng, 1T, 4R

Zhang, Meiju, 3M

Zhang, Ne-jie, 32

Zhang, Peng, 2l

Zhang, Pengfei, 1L, 1R

Zhang, Rui, OD

Zhang, Rui, 4S

Zhang, Run, 28

Zhang, Shaofeng, $2 \mathrm{H}$

Zhang, Shidai, 25

Zhang, Shixin, 2L

Zhang, Shuangya, 1A

Zhang, Song, $1 \mathrm{M}$

Zhang, Su, 2C

Zhang, Tianheng, 42

Zhang, Tieying, 2D

Zhang, Ting, 4B

Zhang, Tonggang, OF

Zhang, Weihong, 0

Zhang, Wentao, 09

Zhang, Xin, 4L

Zhang, Xunbiao, 01

Zhang, Y. Z., 35

Zhang, Yabin, 00

Zhang, Yan, 15

Zhang, Yan, $2 X$

Zhang, Yihui, 1L, 1R

Zhang, Zhen, 3A

Zhang, Zhi Min, $2 M$

Zhang, Zhonghua, 10

Zhang, Zhongyu, 16, $1 \mathrm{~F}$

Zhang, Zili, 2X

Zhao, Bin, 2E

Zhao, Bo, 3A

Zhao, Dongsheng, $1 G$

Zhao, Guolong, 3A

Zhao, Heng, OP

Zhao, Hong, 37

Zhao, Hui, OT

Zhao, Huijie, OQ, OR

Zhao, Huining, 28

Zhao, Jianting, 4L

Zhao, Jun, 27

Zhao, Liping, $\mathrm{OH}$

Zhao, Qiancheng, 1K

Zhao, Tiepeng, 20

Zhao, Yinming, $2 \mathrm{~N}, 2 \mathrm{U}$

Zhao, Yue, 48

Zhao, Zhuo, $\mathrm{OZ}$

Zheng, Gang, 2J

Zheng, Huixin, 4K

Zheng, Peng, $1 Y$

Zheng, Yuansong, 3N

Zheng, Zhongpeng, 4T

Zhi, Zhanxin, IY 
Zhong, Wenting, 1U, 26

Zhong, Zhi, 00, 1W

Zhou, Han, IW

Zhou, He, 38, 3Q

Zhou, Hui, OW

Zhou, Jianfei, 16, $1 \mathrm{~F}$

Zhou, Jianmin, $2 \mathrm{~F}$

Zhou, Peng, 38

Zhou, Qian, 05, 0V, 12, 3l, 3P, 3W, 3Y

Zhou, Shengyang, $2 \mathrm{G}$

Zhou, Tong, 4N

Zhou, Weihu, $2 X$

Zhou, Xiangdong, $1 \mathrm{E}$

Zhou, Ye, 32

Zhou, $\mathrm{Yu}, \mathrm{OH}$

Zhou, Zili, 2N, 2U

Zhu, Fan, 2T

Zhu, Ge, 1A

Zhu, Hong, 22, 3Q

Zhu, J., 04

Zhu, Nannan, 3X, 47

Zhu, Xaioyu, 3 Z

Zhu, Zhenmin, $2 \mathrm{R}$

Zou, Limin, 2C, 21

Zuo, Cuilian, OG 


\title{
Conference Committee
}

\author{
Honorary Chairs \\ Guofan Jin, Tsinghua University (China) \\ Tongbao Li, Tongji University (China) \\ Zhonghua Zhang, National Institute of Metrology (China) \\ Jie Gao, Sichuan University (China) \\ Shenghua Ye, Tianjin University (China) \\ Yuri V. Chugui, TDI SIE, Siberian Branch of the Russian Academy of \\ Sciences (Russian Federation) \\ Zhu Li, Huazhong University of Science and Technology (China) \\ Ahmed Abou-zeid, Physikalisch-Technische Bundesanstalt (Germany) \\ Peter Rolfe, Università degli Studi di Genova (Italy)

\section{Conference Chair} \\ Jiubin Tan, Harbin Institute of Technology (China)

\section{Conference Co-chairs} \\ Tony Wilson, University of Oxford (United Kingdom) \\ Harald Bosse, Physikalisch-Technische Bundesanstalt (Germany) \\ Min Gu, Swinburne University of Technology (Australia) \\ Kuang-Chao Fan, National Taiwan University (Taiwan, China) \\ Wei Gao, Tohoku University (Japan) \\ Seung-Woo Kim, Korea Advanced Institute of Science and Technology \\ (Korea, Republic of) \\ Program Committee Chair \\ Jiubin Tan, Harbin Institute of Technology (China) \\ Program Committee Co-chairs
}

Ahmed Abou-zeid, Physikalisch-Technische Bundesanstalt (Germany)

Igor A. Konyakhin, Saint-Petersburg State University of Information Technologies, Mechanics and Optics (Russian Federation)

Liang-Chia Chen, National Taiwan University (Taiwan, China)

Yongsheng Gao, Hong Kong University of Science and Technology

(Hong Kong, China) 
Program Committee Members

Harald Bosse, Physikalisch-Technische Bundesanstalt (Germany)

Frank Härtig, Physikalisch-Technische Bundesanstalt (Germany)

Min Gu, Swinburne University of Technology (Australia)

Wei Gao, Tohoku University (Japan)

Richard Leach, National Physical Laboratory (United Kingdom)

Christan Rothleitner, Physikalisch-Technische Bundesanstalt (Germany)

Fu-Jen Kao, National Yang-Ming University (Taiwan, China)

M. Selim Ünlü, Boston University (United States)

Martin Booth, University of Oxford (United Kingdom)

Michael Krystek, Physikalisch-Technische Bundesanstalt (Germany)

Ming Chang, Chung Yuan Christian University (Taiwan, China)

Shuang Zhang, University of Birmingham (United Kingdom)

Shulian Zhang, Tsinghua University (China)

Mr. Aiwen Ma, Chinese Society for Measurement (CSM) (China)

Mr. Youhua Wu, China Instrument and Control Society (CIS) (China)

Guobiao Wang, National Natural Science Foundation of China (China)

Jens Flügge, Physikalisch-Technische Bundesanstalt (Germany)

Jiwen Cui, Harbin Institute of Technology (China)

Organizing Committee Chair

Jian Liu, Harbin Institute of Technology (China)

\section{Organizing Committee Co-chairs}

Zhengtao Yu, Kunming University of Science and Technology (China)

Jing Na, Kunming University of Science and Technology (China)

Xinghua Qu, Tianjin University (China)

Lijiang Zeng, Tsinghua University (China)

Zhaoyao Shi, Beijing University of Technology (China)

Qibo Feng, Beijing Jiaotong University (China)

Weihu Zhou, Academy of Opto-Electronics, Chinese Academy of

Sciences (China)

Lianqing Zhu, Beijing Information Science \& Technology University (China)

Yinxiao Miao, Beijing Aerospace Institute of Metrology and Measurement

(China)

\section{Organizing Committee Members}

Chuan Li, Kunming University of Science and Technology (China)

Xing Wu, Kunming University of Science and Technology (China)

Lihua Wang, Kunming University of Science and Technology (China)

Yu Guo, Kunming University of Science and Technology (China)

Jiaru Chu, University of Science and Technology of China (China)

Tianquan Fan, Institute of Optics and Electronics, Chinese Academy of

Sciences (China) 
Zili Zhou, Chinese Aeronautical Establishment (China)

Yuchi Lin, Tianjin University (China)

Hua Ai, Changchun Institute of Optics Fine Mechanics and Physics, Chinese Academy of Sciences (China)

Yinhan Gao, Jilin University (China)

Junjie Guo, Xi'an Jiaotong University (China)

Donglin Peng, Chongqing University of Technology (China)

Guozheng Yan, Shanghai Jiaotong University (China)

Linna Zhang, Zhengzhou University (China)

Ying Xu, Guangdong University of Technology (China)

Dengxin Hua, Xi'an University of Technology (China)

Jianhua Wang, Xi'an Technological University (China)

Xiaoyang Yu, Harbin University of Science and Technology (China)

Yueke Wang, National University of Defense Technology (China)

Yan Li, Tsinghua University (China)

Xiangzhao Wang, Shanghai Institute of Optics and Fine Mechanics (China)

Guoyu Zhang, Changchun University of Science and Technology (China)

Yongying Yang, Zhejiang University (China)

Zhongyu Wang, Beihang University (China)

Qun Hao, Beijing Institute of Technology (China)

Hui Zhao, Shanghai Jiaotong University (China)

Xiaodong Wang, Dalian University of Technology (China)

Weiqian Zhao, Beijing Institute of Technology (China)

Tiehua Ma, North University of China (China)

Mingxing Jiao, Xi'an University of Technology (China)

Yingjie Yu, Shanghai University (China)

Rongsheng Lu, Hefei University of Technology (China)

Yong Xu, Changcheng Institute of Metrology \& Measurement (China)

Yongrui Zhao, China University of Petroleum (China)

Zhihong Liu, Beijing Oriental Institute of Measurement and Test (China)

Liandong Yu, Hefei University of Technology (China)

Shiyuan Liu, Huazhong University of Science and Technology (China)

Changcai Cui, Huaqiao University (China)

Zi Xue, National Institute of Metrology (China)

Jun Han, Xi'an Technological University (China)

Benyong Chen, Zhejiang Sci-Tech University (China)

Jigui Zhu, Tianjin University (China)

Yong Zhu, Chongqing University (China)

Fajie Duan, Tianjin University (China)

Jun Yang, National University of Defense Technology (China)

Yajun Liang, Beijing Aerospace Institute for Metrology and Measurement Technology (China)

Shuming Yang, Xi'an Jiaotong University (China)

Pengcheng Hu, Harbin Institute of Technology (China)

Junning Cui, Harbin Institute of Technology (China)

Huijie Zhao, Beihang University (China) 
Sen Han, University of Shanghai for Science and Technology (China)

Qing He, National Institute of Metrology (China)

Wenlong Lu, Huazhong University of Science and Technology (China)

Yang Liu, Harbin Institute of Technology (China)

Yunfeng Lu, National Institute of Metrology (China)

Secretaries-General

Junning Cui, Harbin Institute of Technology (China)

Doctor Jie Lin, Harbin Institute of Technology (China)

Pengcheng Hu, Harbin Institute of Technology (China)

Plenary Presentation Chairs

Tony Wilson, University of Oxford (United Kingdom)

Fu-Jen Kao, National Yang-Ming University (Taiwan, China)

Seung-Woo Kim, Korea Advanced Institute of Science and Technology

(Korea, Republic of)

Nigel M. Jennett, Coventry University (United Kingdom)

Session Chairs

1 Instrumentation Theory and Methodology I

Christian Rothleitner, Physikalisch-Technische Bundesanstalt (Germany)

Shuming Yang, Xi'an Jiaotong University (China)

2 Measurement for Precision and Ultra-Precision Machining

Jie Zhang, University of Bristol (United Kingdom)

Yan Zhang, Capital Normal University (China)

3 Novel Instrument and Measurement System I

Michael Krystek, Physikalisch-Technische Bundesanstalt (Germany)

Jiwen Cui, Harbin Institute of Technology (China)

$4 \quad$ Novel Instrument and Measurement System II

Olivier Beuf, CNRS (France)

Weihu Zhou, Academy of Opto-Electronics, Chinese Academy of

Sciences (China)

$5 \quad$ Modern Optics and Instruments for Precision Measurement I

M. Selim Ünlü, Boston University (United States)

Xiaojun Liu, Huazhong University of Science and Technology (China)

6 Sensors, Actuators and Application I

Nigel M. Jennett, Coventry University (United Kingdom)

Qibo Feng, Beijing Jiaotong University (China) 
$7 \quad$ Micro and Nano Metrology, Macro Metrology

Seung-Woo Kim, Korea Advanced Institute of Science and Technology (Korea, Republic of)

Pengcheng Hu, Harbin Institute of Technology (China)

8 Laser Measurement Techniques and Instruments I

Igor Konyakhin, Saint-Petersburg State University of Information

Technologies, Mechanics and Optics (Russian Federation)

Xiaodong Wang, Dalian University of Technology (China)

9 Instrumentation Theory and Methodology II

Ling Hao, National Physical Laboratory (United Kingdom)

Liandong Yu, Hefei University of Technology (China)

10 Instrument and Measurement System Calibration I

Yongsheng Gao, Hong Kong University of Science and Technology (Hong Kong, China)

Zi Xue, National Institute of Metrology (China)

11 Signal Processing and Image Processing

Jian Liu, Harbin Institute of Technology (China)

Xinghui Li, Research Institute of Tsinghua University in Shenzhen (China)

12 Sensors, Actuators and Application II

Shuang Zhang, University of Birmingham (United Kingdom)

Yongying Yang, Zhejiang University (China)

13 Laser Measurement Techniques and Instruments II

Mingxing Jiao, Xi'an University of Technology (China)

Donglin Peng, Chongqing University of Technology (China)

14 Novel Instrument and Measurement System III

Benyong Chen, Zhejiang Sci-Tech University (China)

Lianqing Zhu, Beijing Information Science \& Technology University (China)

15 Modern Optics and Instruments for Precision Measurement II

Lijiang Zeng, Tsinghua University (China)

Zhaoyao Shi, Beijing University of Technology (China)

16 Instrument and Measurement System Calibration II

Steven T. Cundiff, University of Michigan (United States)

Qun Hao, Beijing Institute of Technology (China) 
Proc. of SPIE Vol. 11053 1105301-24

Downloaded From: https://www.spiedigitallibrary.org/conference-proceedings-of-spie on 25 Apr 2023 Terms of Use: https://www.spiedigitallibrary.org/terms-of-use 


\title{
Plenary Presentation Biographies
}

\section{Optical fiber sensors for industrial applications}

\author{
9:10-9:50, 9 August 2018 \\ Session Chair: Tony Wilson, Univ. of Oxford (United Kingdom) \\ Prof. Kenneth Grattan

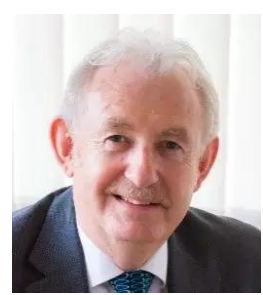 \\ President of the International Measurement Confederation \\ (IMEKO) \\ Dean, City Graduate School \\ Royal Academy of Engineering - George Daniels Professor of \\ Scientific Instrumentation \\ City University of London (United Kingdom)
}

Professor Grattan graduated in Physics from Queen's University Belfast with a BSc (First Class Honors) in 1974 and a PhD in Laser Physics. His research involved the use of laser-probe techniques for measurements on potential new laser systems. Following Queen's, in 1978 he became a Research Fellow at Imperial College of Science and Technology, sponsored by the Rutherford Laboratory to work on advanced photolytic drivers for novel laser systems. This involved detailed measurements of the characteristics and properties of novel laser species and a range of materials involved in systems calibration. In 1983 he joined City University as a "new blood" Lecturer in Physics, being appointed Professor of Measurement and Instrumentation in 1991 and Head of the Department of Electrical, Electronic and Information Engineering. He was appointed Dean of the Schools of Engineering \& Mathematical Sciences and of Informatics in 2008, serving until 2012 when he became Dean of the newly formed City Graduate School. His research interests include the use of fiber optic and optical systems in the measurement of a range of physical and chemical parameters. The work has been sponsored by a number of organizations including EPSRC, the EU, private industry and venture capital and he holds a number of patents for his work with industry. He obtained a DSc from City University in 1992 for his sensor work.

Professor Grattan is extensively involved with the work of the professional bodies having been Chairman of the Science, Education and Technology of the Institution of Electrical Engineers, the Applied Optics Division of the Institute of Physics and he was President of the Institute of Measurement and Control during the year 2000. He was awarded the Callendar Medal of the Institute of Measurement and Control in 1992, the Hartley Medal of the same Institution in 2015 and the Honeywell Prize for work published in the Institute's journal as well the Institute of Physics Applied Optics Divisional Prize in 2010. Professor Grattan had been Deputy Editor of the Journal Measurement Science and Technology for several years and currently serves on the Editorial Board of several major journals in his field in the USA and Europe. In January 2001 he was appointed Editor of the IMEKO Journal "Measurement" and now is Editor Emeritus of the Journal. After many years senving on their General Council, he was appointed the President of the 
International Measurement Confederation (IMEKO) in 2015. He is the author and co-author of about 1300 publications in major international joumals and at conferences and is the co-editor (with Professor B T Meggitt) of a five volumes topical series on Optical Fiber Sensor Technology. Professor Grattan was Dean of the School of Engineering \& Mathematical Sciences and also Dean of the School of Informatics at City University from 2008 to 2012 and in that year was appointed as the Inaugural Dean of the new City Graduate School at the University. 


\title{
Miniature two-photon microscopy for brain imaging in freely behaving animals
}

\author{
9:50-10:30, 9 August 2018 \\ Session Chair: Tony Wilson, Univ. of Oxford (United Kingdom)
}

Prof. Heping Cheng

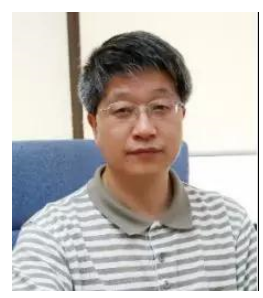

Leader of Institute of Molecular Medicine, Peking University

Fellow of the Chinese Academy of Sciences (China)

Professor Heping (Peace) Cheng received his bachelor and master degrees in applied mathematics \& mechanics and biomedical engineering, with physiology as his minor, from Peking University, China. Upon graduation, he served as a junior faculty member in the Department of Electrical Engineering at the same university before earning his Ph.D. degree in physiology in 1995 from the University of Maryland at Baltimore. He then joined the NIH Intramural Research Program as a senior staff fellow, was selected as a tenure-track investigator in 1998 and became the head of the Ca2+ Signaling Section in the Laboratory of Cardiovascular Science, National Institute of Aging, NIH. He was promoted to senior investigator in 2004. He is now a senior investigator heading the Laboratory of $\mathrm{Ca} 2+$ Signaling \& Mitochondrial Biomedicine in the Institute of Molecular Medicine at Peking University. He was elected to the Chinese National Academy of Sciences in 2013. Co-discovering "Ca2+ sparks" in 1993 and mitochondrial "superoxide flashes" in 2008, he strives to resolve elemental physiological signals in the pursuit of principles of cell signaling. Currently he is engaged in developing novel imaging technology for reverse engineering of brain information processing. 


\title{
Comb-based multidimensional coherent spectroscopy
}

\author{
10:45-11:25, 9 August 2018 \\ Session Chair: Fu-Jen Kao, National Yang-Ming Univ. \\ (Taiwan, China)
}

\section{Prof. Steven Cundiff}

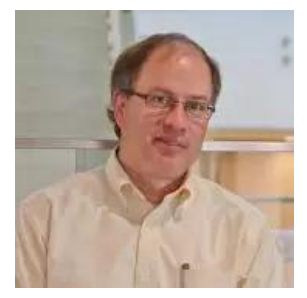

Fellow Adjoint of JILA. Harrison M. Randall Collegiate Professor of Physics, University of Michigan, Ann Arbor (United States)

Fellow of the IEEE, Fellow of the APS, Fellow of the OSA, OSA Meggers Award, Humboldt Research Award

Professor Cundiff and his research group work on several aspects of ultrafast optics. One area involves generating and controlling ultrashort pulses, which, of course, provides the foundation for the field of ultrafast optics. However, the group is primarily interested in using ultrashort light pulses for a variety of scientific applications. A natural application is to use the very short duration of the pulses to study processes that occur on similar timescales, which is generally known as ultrafast spectroscopy. Ultrafast spectroscopy not only gives dynamical information, but it also provides information about the fundamentals of how light interacts with matter. One type of ultrafast spectroscopy, known as optical multidimensional coherent spectroscopy, has been developed over the last decade as has proven to be very powerful. The Cundiff group uses ultrafast spectroscopy, including multidimensional coherent spectroscopy, to study a range of system including semiconductors, semiconductor nanostructures and atomic vapors. 


\title{
A novel high-precision mass measurement device for the new kilogram
}

\author{
$11: 25-12: 05,9$ August 2018 \\ Session Chair: Fu-Jen Kao, National Yang-Ming Univ. \\ (Taiwan, China)
}

Dr. Christan Rothleitner

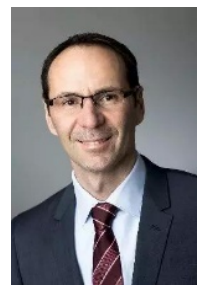

Leading scientist of the group mass metrology for Planck balances, Physikalisch-Technische Bundesanstaldt (PTB) (Germany) Member of German Physical Society DPG and American Physical Society

Dr. Christian Rothleitner studied physics in Germany, Italy and Venezuela. He received his PhD in experimental physics at the Max Planck Institute for the Science of Light, in Germany, about the development of two free-fall absolute gravimeters in the group of Prof. Lijun Wang (now at Tsinghua University, China). After he received his PhD he made a postdoctorate at the University of Luxembourg where he developed a free-fall experiment to measure the Newtonian constant of gravitation. Thereafter, he joined the German national metrology institute, the Physikalisch-Technische Bundesanstaldt (PTB), where he gained several years of experience in length metrology with a special focus on computed tomography. Now he is the leading scientist of the group mass metrology for Planck balances at PTB. In this position he is responsible for developing a high-precision weighing instrument that will allow to make primary realizations of the SI unit kilogram after its re-definition by end of 2018. This is done in collaboration with the Technical University of Ilmenau where Dr. Rothleitner is also doing his 'habilitation', a qualification as a lecturer. Dr. Rothleitner published more than 30 scientific articles in international peer reviewed journals. He is member of the German physical society DPG and of the American physical society APS. 


\title{
Time resolved imaging with stimulated emission in pump-probe microscopy
}

\author{
13:30-14:10, 9 August 2018 \\ Session Chair: Seung-Woo Kim, Korea Advanced Institute of \\ Science and Technology (Korea, Republic of)
}

Prof. Fu-Jen Kao

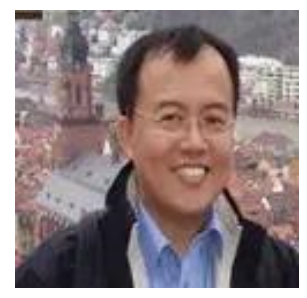

Professor, Institute of Biophotonics, National Yang-Ming University (Taiwan, China) (2004-)

Association of Asia Pacific Physical Societies (2016-)

Professor Fu-Jen Gao is now in Institute of Biophotonics, National Yang-Ming University since 2004 and also the association of Asia Pacific Physical Societies. He was the president of Physics Society of ROC (2012-2014), vice president of Physics Society of ROC (2012-2014), associated Dean of Office of Research \& Development, NYMU (20062011), and also the director, Institute of Biophotonics, NYMU (2004-2011). His research interests are in the field of Stimulated emission based pump-probe microscopy, 4channel Stokes vector resolved SH polarization microscopy and biomedical optical instrument for endoscopy. During his academic career, the long working distance fluorescence and lifetime measurement via stimulated emission, and laser illumination for endoscopy are the two research highlights.

In the field of "long working distance fluorescence and lifetime measurement via stimulated emission", Prof Gao and his team are focusing on the unique aspect of spatial coherence as a result of stimulated emission, which is utilized for long distance fluorescence detection and lifetime imaging. In contrast with the case of spontaneous emission, high numerical aperture optics is not required to collect the stimulated emission signal efficiently.

Meanwhile, in the field of "Laser illumination for endoscopy", Prof Gao's team have successfully established a novel ultra-compact endoscopic imaging system, which uses a miniature CMOS sensor (O.D. $<1.0 \mathrm{~mm}$ ) and a few multimode fiber for light delivery. Critically, the illumination is realized by coupling the output of a supercontinuum or RGB laser into the fiber. In this way, very high brightness is possible with extremely small footprint on the illumination part. As a result, the overall diameter $(<1.2 \mathrm{~mm})$ of the endoscope can be much smaller than the currently used models. 


\title{
Size matters! Understanding and exploiting the length-scale dependence of material properties and nano/micro-scale measurements
}

\author{
14:10-14:50, 9 August 2018 \\ Session Chair: Seung-Woo Kim, Korea Advanced Institute of \\ Science and Technology (Korea, Republic of)
}

Prof. Nigel M. Jennett

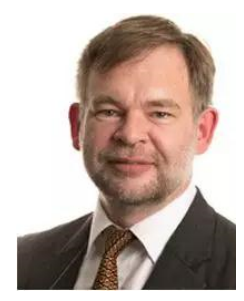

Professor of Materials, Mechanics and Measurement at Coventry University *United Kingdom)

Chair of the BSI Indentation Hardness Committee

Professor Nigel M. Jennett BSC (Hons) (Physics), PhD (Physics), CSci CPhys MinstP has over 25 years' experience of fabrication and characterization of nano-structured materials and 20 years' developing nano-mechanical test methods. He is: Professor of Materials, Mechanics and Measurement at Coventry University, visiting Professor of Engineering at Leicester University, Associate Editor of Philosophical Magazine (and Philos. Mag. Letters), international chair of VAMAS Technical Working Area 22 'Mechanical properties measurement of thin films and coatings', UK technical expert on the CIPM consultative committee hardness working group (CCM-WGH), chairs the BSI indentation hardness committee, leads the UK delegation for ISO working groups drafting standards for indentation-based test methods. Nigel has also served two terms (six years) on the European Commission Certification Advisory Panel for Physical and Physicochemical Properties.

Nigel studied Physics at Bristol University (Physics Laboratory prize in 1984 and 1986, and the Raychem prize in 1985). He spent six years researching magnetic multilayers (1990 PhD, 1991 Chartered Physicist), before moving to NPL (1992) to develop traceable Scanned Probe Microscopy and nano-mechanical measurements. In 1998 he created his own research group focused on surfaces, coatings and nano-mechanics and was awarded a Glazebrook Fellowship in 2003 and the NPL Rayleigh award in 2010. Nigel is an experienced leader of projects (Government, Industry and European Commission), and is a regular invited speaker at international conferences. 


\title{
Innovative techniques for contrast, spectrometric and viscoelastic measurements in small animal MRI
}

\author{
14:50-15:30, 9 August 2018 \\ Session Chair: Seung-Woo Kim, Korea Advanced Institute of \\ Science and Technology (Korea, Republic of)
}

Prof. Olivier Beuf

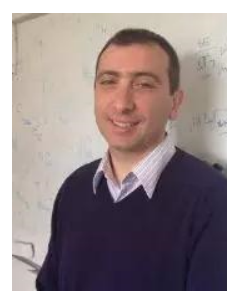

Senior CNRS Research Scientist

Team Leader "NMR and optics: From measure to biomarker"

Director of the CREATIS Lab, CNRS, INSERM (France)

Dr. Olivier Beuf is the senior CNRS research scientist in France. He obtained his PH.D in physics from Université Claude Bernard Lyon 1 in 1998. Dr. Beuf has widely research interests in the field of MR imaging, RF coils, multi-parameters quantitative imaging, liver analysis, cartilage ultra-structure and morphology, and so on.

He published more than 80 peer reviewed international journal articles and 8 book chapters. His research works are 1105 citations in WOS and the h-index is as high as 19. Meanwhile, the transfer of technology are 3 patents. Dr. Beuf is also the supervisor of $17 \mathrm{PhD}$ students (14 defended and 3 still supervised). Dr. Beuf is the chairman of the "journées scientifiques sur les nouvelles méthodologies en imagerie du vivant", Lyon, France (300 delegates). He is the distinguished reviewer of Journal of Magnetic Resonance Imaging (2011 and 2014) and Magna Cum Laude Merit Award of the 30th Meeting of the International Society for Magnetic Resonance in Medicine (2012). 


\title{
Interferometric microscopy for detection and visualization of biological nanoparticles
}

\author{
15:45-16:25, 9 August 2018 \\ Session Chair: Nigel M. Jennett, Coventry Univ. \\ (United Kingdom)
}

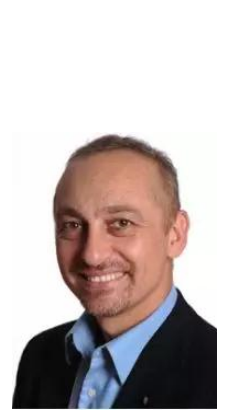

Prof. M. Selim Ünlü

\author{
Distinguished Professor of Engineering appointed in electrical and \\ computer engineering, biomedical engineering, physics, and \\ graduate medical sciences. Boston University (United States) \\ IEEE Fellow and OSA Fellow \\ Editor-in-Chief for IEEE Journal of Quantum Electronics \\ Contact Information: selim@bu.edu, www.bu.edu/OCN
}

Professor M. Selim Ünlü received the B.S. degree from the Middle East Technical University, Ankara, Turkey, in 1986, and the M.S.E.E. (1988) and Ph.D. (1992) degrees from the University of Illinois at Urbana-Champaign, all in electrical engineering. Since 1992, he has been a professor at Boston University. He is currently a Distinguished Professor of Engineering appointed in electrical and computer engineering, biomedical engineering, physics, and graduate medical sciences. He has also served as the Associate Dean for Research and Graduate Programs in engineering. His research interests are in the areas of nanophotonics and biophotonics focusing on high-resolution solid immersion lens microscopy of integrated circuits and development of biological detection and imaging techniques, particularly in high-throughput digital biosensors based on detection of individual nanoparticles and viruses.

Dr. Ünlü was the recipient of the NSF CAREER and ONR Young Investigator Awards in 1996. He has been selected as a Photonics Society Distinguished Lecturer for 2005-2007 and Australian Research Council Nanotechnology Network (ARCNN) Distinguished Lecturer for 2007. He has been elevated to IEEE Fellow rank in 2007 for his "contributions to optoelectronic devices" and OSA Fellow rank in 2017 for his "for pioneering contributions in utilization of optical interference in enhanced photodetectors and biological sensing and imaging." In 2008, he was awarded the Science Award by the Turkish Scientific Foundation. His professional service includes serving as the chair of the Annual Meeting for IEEE Photonics Society and Editor-in-Chief for IEEE Journal of Quantum Electronics. 


\title{
Plasmonics: Exotic nanophotonics beyond the limits
}

\author{
16:25-17:05, 9 August 2018 \\ Session Chair: Nigel M. Jennett, Coventry Univ. \\ (United Kingdom)
}

\section{Prof. Satoshi Kawata}

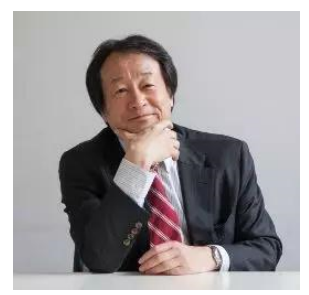

\author{
Professor Emeritus, Osaka University (Japan) \\ Honorary Scientist RIKEN (Japan) \\ Osaka University, Suita (Japan) \\ Office: P3-300, Photonics Center \\ Email:kawata@ap.eng.osaka-u.ac.jp
}

Professor Satoshi Kawata is now Professor Emeritus at Osaka University and Honorary Scientist of RIKEN. He is the founder and the Chairman of the Board of Nanophoton Corp. He is a Fellow of OSA, IOP, SPIE, and JSAP.

Satoshi Kawata received his BSC, MsC, and PhD all in Applied Physics in 1974, 76, and 79, respectively, from Osaka University. After the experience of postdoctral fellow of JSPS, he spent two years in University of California, at Irvine as a Research Associate. He joined Osaka University as a faculty member in 1981 and was promoted to Professor of Applied Physics in 1993, and then Distinguished Professor in 2013. In 2002, he joined RIKEN as a Chief Scientist as Head of Nanophotonics Laboratory until his retirement in 2012, and Team Leader of RIKEN until 2015.

Professor Kawata is now the Professor Emeritus of Osaka University and Honorary Scientist of RIKEN. He has served as the President of JSAP (Japan Society of Applied Physics) from 2014 to 2016, and the President of Spectroscopical Society of Japan from 2007 to 2008, the Editor of Optics Communications from 2000 to 2009.

$\mathrm{He}$ is one of the pioneers in near field optics (the inventor of tip-enhanced near-field microscopy), three-dimensional microscopy (laser CT microscopy, 3D optical data storage), plasmonics (SPR sensors, plasmon holography, plasmon laser, plasmonic microscopy), two-photon engineering (two-photon polymerization, two-photon isomerization, two-photon photorefraction, two-photon SPP, etc), bio-imaging, and signal recovery. The "8-micron bull" fabricated with his invented two-photon technology has been awarded in Guinness World Record Book 2004 Edition. 


\title{
Drive operational excellence through intelligent quality
}

\author{
17:05-17:45, 9 August 2018 \\ Session Chair: Nigel M. Jennett, Coventry Univ. \\ (United Kingdom)
}

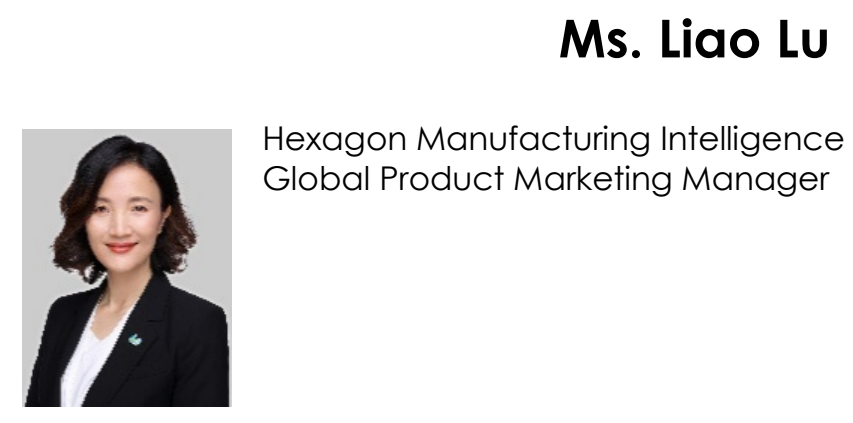

Ms. Liao Lu is now Hexagon Manufacturing Intelligence Global Product Marketing Manager. She is also HxGN SMART Quality Product Marketing Manager. With more than 20 years of industrial experience in precision measurement, Ms. Liao Lu has extensive market knowledge and internationalized view on measuring technology and customer application, and has made important contributions to the application and popularization of advanced measuring technology. She received her MSc degrees in Precision Measuring Technology from Tianjin University in 2003. 
Proc. of SPIE Vol. 11053 1105301-36

Downloaded From: https://www.spiedigitallibrary.org/conference-proceedings-of-spie on 25 Apr 2023 Terms of Use: https://www.spiedigitallibrary.org/terms-of-use 


\section{Introduction}

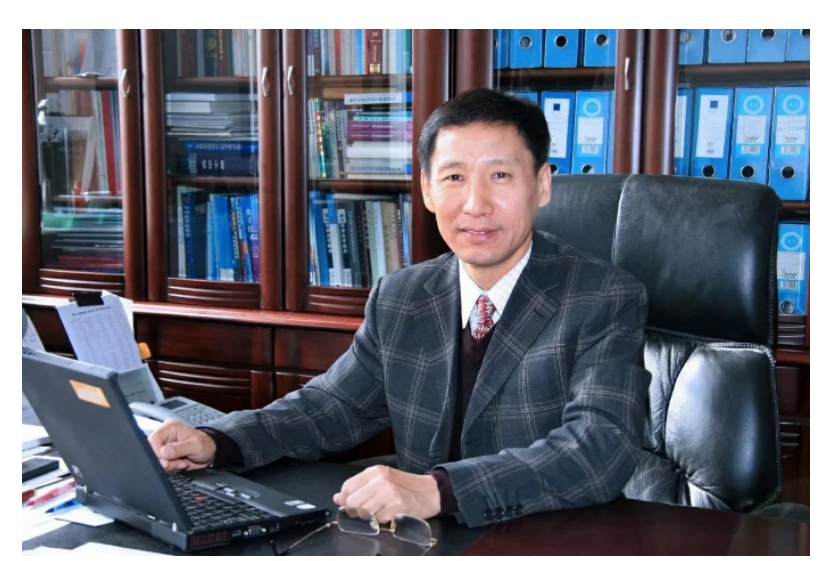

The International Symposium on Precision Engineering Measurements and Instrumentation (ISPEMI) is an international symposium held every other year since 1999, in different cities of China with English as its working language. ISPEMI 2018 is the 10th ISPEMI, and was held 8-10 August 2018, in Kunming, Yunnan Province. It is sponsored by the International Committee on Measurements and Instrumentation (ICMI), National Natural Science Foundation of China (NSFC), Chinese Society for Measurement (CSM), and China Instrument and Control Society (CIS) and organized by Harbin Institute of Technology (HIT), International Committee on Measurements and Instrumentation (ICMI), and Instrumentation Committee of CSM (IC-CSM), in cooperation with Kunming University of Science and Technology, Beijing Information Science and Technology University, Hefei University of Technology, and the Electricity and Magnetism Committee of CSM.

The purpose of ISPEMI 2018 is to bring together young and senior researchers and engineers working in the fields of precision engineering measurements and instrumentation all over the world and provide an international technical forum for them to present their research and develop their knowledge of the recent advances on this particular aspect.

The theme of this conference is measurement and instrument for new industrial era. The morning glow of a new round of a science and technology revolution has already emerged in recent years. New technologies, such as a new generation of internet, big data, cloud computation, three-dimensional printing, artificial intelligence, quantum communication, quantum computation, and quantum measurement are developing fast. They will bring about a new generation of industrial revolution. Intelligent manufacturing marked by intelligent equipment and intelligent factory has already appeared. At the same time, International Measurement Organization has implemented the redefinition of seven basic quantities. All these put forward a significant challenge for precision engineering measurement and instrument technologies. How do measurement and instrument play guiding and supporting roles in this science and technology revolution and industrial revolution? This is the historic mission all the measurement and instrument experts and scientists in this new era are now facing. We are going to continue the discussion on this historic issue at the conference. 
At ISPEMI 2018, ten plenary speakers from the United Kingdom, Germany, the United States, France, Japan, and China, made excellent presentations. From the 270 received manuscripts, we accepted only 104 papers for oral and 139 papers for poster presentation. Professor Tony Wilson concluded that the conference was very successful in terms of subject and organization.

While the Proceedings of ISPEMI 2018 is now ready for delegates, we would like to thank National Natural Science Foundation of China (NSFC), International Committee on Measurements and Instrumentation (ICMI), Chinese Society for Measurement (CSM), China Instrument and Control Society (CIS), Harbin Institute of Technology (HIT), SPIE, Kunming University of Science and Technology, Beijing Information Science and Technology University, and Hefei University of Technology for their funds and assistance provided. Our thanks go to the procedure and organizing committee members, especially honorary chairman, co-chairmen, and plenary speakers, Prof. Kenneth Grattan, Prof. Heping Cheng, Prof. Steven Cundiff, Dr. Christian Rothleitner, Prof. Fu-Jen Kao, Prof. Nigel M. Jennett, Prof. Olivier Beuf, Prof. M. Selim Ünlü, Prof. Satoshi Kawata, and Dr. Liao Lu for their efforts to make the meeting fruitful and successful. Our special thanks go to SPIE for its efforts to enable us to do these things so well. It was also declared the ISPEMI 2020 will be held in Guilin in Guangxi Province. See you again then.

Jiunin Tan 